\title{
Some properties of IIR power-symmetric filters
}

\author{
P. P. Vaidyanathan \\ Dept. of Electrical Engineering \\ California Institute of Technology \\ Pasadena, CA 91125 \\ Email: ppvnath@systems.caltech.edu
}

\begin{abstract}
Power-symmetric IIR filters have in the past been used in two-channel filter banks. If appropriately designed, such filters have allpass polyphase components, and this induces useful properties in the filter bank. For example, IIR orthonormal filter banks have in the past been designed in this way, and generate orthonormal basis functions. In this paper we study some theoretical properties of IIR power symmetric filters in a more general perspective. This includes the derivation of a general analytical form, and a study of pole locations. ${ }^{1}$
\end{abstract}

Index terms: Power symmetric filters, filter banks, IIR filters, orthogonal waveforms.

\section{INTRODUCTION}

In the early days of filter bank theory, a particular class of IIR two channel filter banks was proposed by a number of authors, in which the two polyphase components were IIR allpass filters [4], [6], [7]. The analysis filters $[G(z), H(z)]$ in this system have the form

$$
G(z)=\frac{a_{0}\left(z^{2}\right)+z^{-1} a_{1}\left(z^{2}\right)}{2}
$$

and $H(z)=G(-z)$, where $a_{0}(z)$ and $a_{1}(z)$ are causal stable allpass filters. The synthesis filters can be chosen to have perfect reconstruction, in which case they are noncausal stable filters [4]. More commonly however, they are chosen such that aliasing and magnitude distortion are eliminated, the remaining phase distortion being acceptable in certain applications such as speech coding [5]. This system is described in considerable detail in Chap. 5 of [5], and it is well-known that Butterworth and elliptic filters can be represented as in Eq. (1) if their specifications are appropriately constrained.

A filter which has the form (1) satisfies the property

$$
\widetilde{G}(z) G(z)+\widetilde{G}(-z) G(-z)=1
$$

where $\widetilde{G}(z)=G^{*}\left(1 / z^{*}\right)$. This is called the power-symmetric property. The name comes from the fact that if $G(z)$ is lowpass with real coefficients then $\left|G\left(e^{j \omega}\right)\right|^{2}$ has a certain symmetry with respect to $\omega=\pi / 2$ (e.g., its passband and stopband ripple sizes are identical, as demonstrated later in Fig. 2(a)). Power symmetry induces useful properties in the

\footnotetext{
${ }^{1}$ Work supported in parts by the Office of Naval Research grant
} N00014-06-1-0011 and the California Institute of Technology. filter bank. For example, IIR orthonormal filter banks have in the past been designed in this way, and generate orthonormal basis functions.

Not all power-symmetric filters can be expressed as in Eq. (1), because the form (1) also implies that the numerator $P(z)$ of $G(z)$ satisfies Hermitian symmetry, that is, $p_{N-n}^{*}=$ $e^{j \epsilon} p_{n}$, for integer $N$ and real $\epsilon$. For example in the real coefficient lowpass case $P(z)$ is symmetric $\left(p_{N-n}=p_{n}\right)$. Conversely, it is shown in [5] (Theorem 5.3.1) that for the real coefficient case if $G(z)$ is power symmetric and has the said numerator symmetry, then it can be expressed as in Eq. (1). More generally a similar result is also true for complexcoefficient filters. Notice that Eq. (1) implies in particular that the denominator of $G(z)$ is a function of $z^{2}$, that is, it only has even powers of $z$.

In this paper we present some deeper properties of powersymmetric filters which have hitherto not been noticed. This includes the general analytical form of such filters (Sec. II), and pole locations (Sec. III). While a limited amount of results on these aspects have been reported earlier (as reviewed in [5]) the material presented here appears to have escaped attention. Given the importance of orthonormal bases in many applications such as data compression, digital communications, and MIMO radar [3], these properties can be of considerable interest.

Unless mentioned otherwise, all notations are as in [5]. The discussions here are not restricted to the case of real coefficients filters.

\section{NECESSARY AND SUFFICIENT CONDITIONS}

We will find it convenient to express $\widetilde{G}(z) G(z)$ in the form

$$
\widetilde{G}(z) G(z)=\frac{1}{1+\widetilde{R}(z) R(z)}
$$

where $R(z)$ is rational. If $G(z)$ is an IIR rational transfer function with $\left|G\left(e^{j \omega}\right)\right| \leq 1$, then we can always write it as in (3). To see this, just observe that

$$
\widetilde{R}(z) R(z)=\frac{1}{\widetilde{G}(z) G(z)}-1
$$

Since $\left|G\left(e^{j \omega}\right)\right| \leq 1$ it follows that

$$
\frac{1}{\left|G\left(e^{j \omega}\right)\right|^{2}}-1 \geq 0
$$


This implies that the right hand side of (4) has rational spectral factors, and we just have to take $R(z)$ to be one such factor. Thus the form Eq. (3) is justified as long as $\left|G\left(e^{j \omega}\right)\right| \leq$ 1. In fact digitial Butterworth, Chebyshev, and Elliptic filters are often introduced in the form (3), and the filter properties are governed by the way in which $R(z)$ is chosen [1], [2]. A natural question is, what are the conditions on the rational form $R(z)$ which make $G(z)$ a power-symmetric filter? This is answered next.

Lemma 1. Power symmetry and $R(z)$. The rational filter $G(z)$ in Eq. (3) satisfies $\widetilde{G}(z) G(z)+\widetilde{G}(-z) G(-z)=1$ if and only if

$$
A_{1}(z) \triangleq
$$

is allpass, or equivalently

$$
A_{2}(z) \triangleq \widetilde{R}(z) R(-z)
$$

is allpass.

Proof. From Eq. (3) we have

$$
\begin{aligned}
& \widetilde{G}(z) G(z)+\widetilde{G}(-z) G(-z) \\
& \quad=\frac{1}{1+\widetilde{R}(z) R(z)}+\frac{1}{1+\widetilde{R}(-z) R(-z)} \\
& \quad=\frac{K(z)+1}{K(z)+\widetilde{R}(z) R(z) \widetilde{R}(-z) R(-z)}
\end{aligned}
$$

where $K(z)=1+\widetilde{R}(z) R(z)+\widetilde{R}(-z) R(-z)$. Clearly this is unity if and only if

$$
\widetilde{R}(z) R(z) \widetilde{R}(-z) R(-z)=1
$$

This condition can be rewritten as $\widetilde{A}_{1}(z) A_{1}(z)=1$ (i.e., $A_{1}(z)$ is allpass) or equivalently as $\widetilde{A}_{2}(z) A_{2}(z)=1$. This proves the desired result.

$$
\nabla \nabla \nabla
$$

\section{II.1 Analytical form for $R(z)$}

Based on the preceding result it is possible to develop a simple analytical form for $R(z)$. More specifically we will show that the results in Lemma 1 can be strengthened considerably as follows:

Theorem 1. Power symmetry and $R(z)$. A rational filter $G(z)$ satisfies $\widetilde{G}(z) G(z)+\widetilde{G}(-z) G(-z)=1$ if and only if we can write

$$
\widetilde{G}(z) G(z)=\frac{1}{1+\widetilde{R}(z) R(z)}
$$

where $R(z)$ has the form

$$
R(z)=\frac{z^{-N} \widetilde{B}(-z)}{B(z)}=\prod_{k=1}^{N}\left(\frac{p_{k}^{*}+z^{-1}}{1-p_{k} z^{-1}}\right)
$$

for some $B(z)=1+\sum_{n=1}^{N} b_{n} z^{-n}$. This condition can also be expressed by saying that $R(z) R(-z)$ is allpass, or equivalently that

$$
\widetilde{R}(z) R(-z)=(-1)^{N}
$$

for all $z . \diamond$

For the real-coefficient case (9) becomes

$$
R(z)=\left(\frac{\sigma+z^{-1}}{1-\sigma z^{-1}}\right)^{\ell} \prod_{k=1}^{L}\left(\frac{d_{k}+c_{k} z^{-1}+z^{-2}}{1-c_{k} z^{-1}+d_{k} z^{-2}}\right)
$$

where $\sigma, c_{k}, d_{k}$ are real, and $N=2 L+\ell$.

Proof of Theorem 1. Assume that $p$ is a pole of the rational function $R(z)$. Since (5) is allpass, it follows [5] that $1 / p^{*}$ is a zero of this function. So $1 / p^{*}$ must be a zero of either $R(z)$ or $R(-z)$. If it is a zero of $R(z)$ then $R(z)$ has the allpass factor $\left(-p^{*}+z^{-1}\right) /\left(1-p z^{-1}\right)$ which does not contribute to the product $\widetilde{R}(z) R(z)$ in Eq. (3). Assuming such redundant allpass factors have been removed from $R(z)$ we see that whenever $p$ is a pole of $R(z)$, then $1 / p^{*}$ is a zero of $R(-z)$, that is $-1 / p^{*}$ is a zero of $R(z)$. Thus $R(z)$ has the form

$$
R(z)=\prod_{k=1}^{N}\left(\frac{p_{k}^{*}+z^{-1}}{1-p_{k} z^{-1}}\right)
$$

There is no need to include a constant unit magnitude scale factor above, because it would cancel in the product $\widetilde{R}(z) R(z)$ in Eq. (3). Equation (12) is readily rewritten as $R(z)=z^{-N} \widetilde{B}(-z) / B(z)$ which means that the allpass filter in (6) simplifies to $A_{2}(z)=$ $\widetilde{R}(z) R(-z)=(-1)^{N}$ indeed.

$\nabla \nabla \nabla$

\section{POLES OF POWER SYMMETRIC FILTERS}

For the case of power-symmetric Butterworth and elliptic filters the poles of $G(z)$ are all on the imaginary axis [5]. This property is a special case arising from the following lemma, which relates the poles of $G(z)$ to those of $R(z)$.

Lemma 2. Poles of power symmetric filters. Let $G(z)$ be a rational, real coefficient, power symmetric filter, so that $R(z)$ has the form (9). Assume that all the poles of $R(z)$ are restricted to be on one side of the imaginary axis (all of them in the left half plane (LHP) or all of them in the right half plane (RHP), with none on the imaginary axis), as demonstrated in Figs. 1(a),(b). Then all the poles of the power symmetric filter $G(z)$ are restricted to be imaginary (see Fig. 1(c)). 


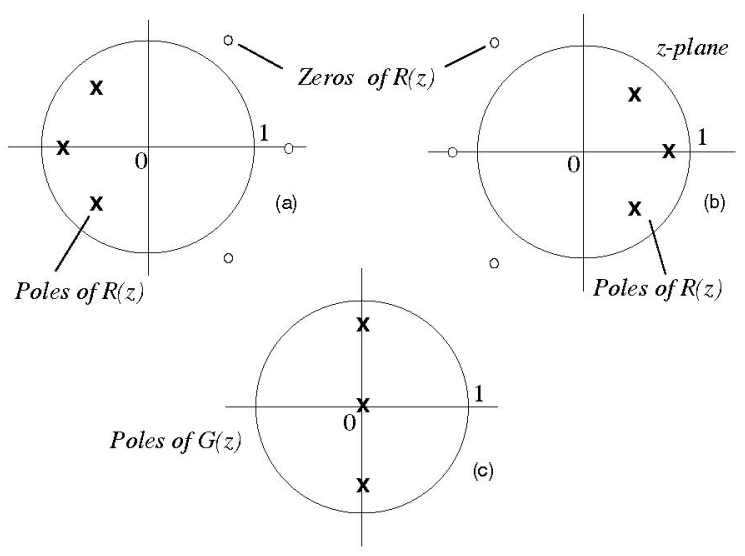

Figure 1. (a)-(c) Pertaining to the poles of power symmetric filters (Lemma 2).

Proof. Since power symmetry of $G(z)$ implies (10), Eq. (8) can be rewritten as

$$
\widetilde{G}(z) G(z)=\frac{1}{1+\frac{(-1)^{N} R(z)}{R(-z)}}
$$

At a pole of $G(z)$ the denominator of the above expression is zero, that is,

$$
\frac{R(z)}{R(-z)}=-(-1)^{N}
$$

In view of the real-coefficient assumption we have $R(-j y)=R^{*}(j y)$ for any real $y$. On the imaginary axis of the $z$-plane we therefore have

$$
\left|\frac{R(j y)}{R(-j y)}\right|=\left|\frac{R(j y)}{R^{*}(j y)}\right|=1
$$

That is, the quantity $R(z) / R(-z)$ has unit-magnitude on the imaginary axis, analogous to a continuous time allpass filter [5]. If $R(z)$ has all its poles on the LHP, then, in view of the modulus property [5], it follows that

$$
\left|\frac{R(z)}{R(-z)}\right| \begin{cases}>1 & \text { in the LHP } \\ <1 & \text { in the RHP } \\ =1 & \text { on the imaginary axis }\end{cases}
$$

The condition (14) can therefore only be satisfied on the imaginary axis. If all the poles of $R(z)$ are on the RHP instead, the argument would be identical. $\quad \nabla \nabla \nabla$

III.1. Cases with poles not on imaginary axis

There are power symmetric filters with poles not on the imaginary axis. For example recall that filters of the from

$$
G(z)=\frac{a_{0}\left(z^{2}\right)+z^{-1} a_{1}\left(z^{2}\right)}{2}
$$

where $a_{0}(z)$ and $a_{1}(z)$ are rational allpass filters, are power symmetric (and the numerators satisfy Hermitian symmetry).
If $a_{k}(z)$ have negative real poles $p_{i}$, then the poles of $G(z)$ are purely imaginary $\left(= \pm j \sqrt{\left|p_{i}\right|}\right.$ ). But if $a_{k}(z)$ have aribtrary poles then $G(z)$ has poles not restricted to the imaginary axis, though they are still symmetric with respect to the imaginary axis. An example would be the power symmetric filter

$$
G(z)=\frac{1}{2}\left(\frac{0.25-z^{-2}}{1-0.25 z^{-2}}\right)+\frac{z^{-1}}{2}\left(\frac{0.04-z^{-2}}{1-0.04 z^{-2}}\right)
$$

The poles of $G(z)$ are real and occur at $z= \pm 0.5$ and $z=$ \pm 0.2 . If, on the other hand, the power symmetric filter is

$$
G(z)=\frac{1}{2}\left(\frac{0.25+z^{-2}}{1+0.25 z^{-2}}\right)+\frac{z^{-1}}{2}\left(\frac{0.04+z^{-2}}{1+0.04 z^{-2}}\right)
$$

then the poles are imaginary and occur at $z= \pm 0.5 j$ and $z= \pm 0.2 j$.

We now consider two special cases of Lemma 2, namely elliptic and Butterworth power-symmetric filters. Chebyshev filters cannot be power symmetric because of inherent lack of symmetry (they have ripples only in one band).

III.2. Elliptic power symmetric filters

For elliptic lowpass filters $R(z)$ has the form [5]

$$
R(z)=\left(\frac{1-z^{-1}}{1+z^{-1}}\right)^{\ell} \prod_{k=1}^{m} \frac{\left(1-z^{-1} e^{j \theta_{k}}\right)\left(1-z^{-1} e^{-j \theta_{k}}\right)}{\left(1-z^{-1} e^{j \omega_{k}}\right)\left(1-z^{-1} e^{-j \omega_{k}}\right)}
$$

where $\ell=0$ for even order filters and unity for odd order filters. Thus $R(z)$ has all its poles and zeros on the unit circle. The poles are at the frequencies $\omega_{k}$ and represents the unit circle zeros of $G(z)$. The zeros are at the frequencies $\theta_{k}$ and represents the passband maxima of $G(z)$ as demonstrated in Fig. 2(a).

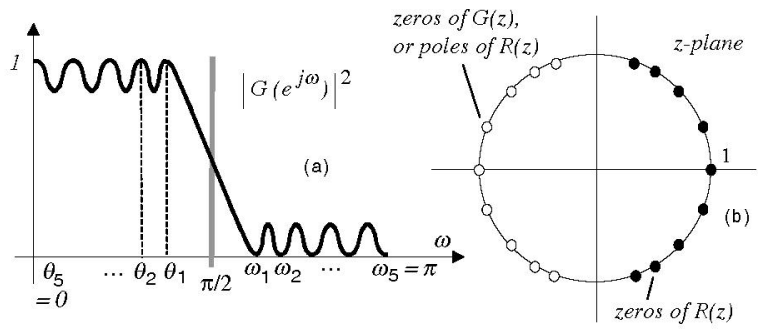

Figure 2. (a) Example of a power symmetric elliptic filter (magnitude square response), and (b) the poles and zeros of the corresponding $R(z)$.

The passband maxima are in the range $0 \leq \omega<\pi / 2$ and the stopband zeros are in the range $\pi / 2<\omega \leq \pi$. Thus the quantities $\omega_{k}$ are in the left half of the $z$-plane and the quantities $\theta_{k}$ are in the right half plane. The poles and zeros of $R(z)$ are therefore in the LHP and RHP respectively as demonstrated in Fig. 2(b). The conditions of Lemma 2 are therefore satisfied, which explains why the poles of these elliptic filters are on the imaginary axis. 


\section{III.3. Butterworth power-symmetric filters}

For IIR Butterworth power-symmetric lowpass filters, all zeros are at $z=-1$. So $R(z)$ has the form

$$
R(z)=\left(\frac{1-z^{-1}}{1+z^{-1}}\right)^{N}
$$

where $N$ is the order of the filter. All poles of $R(z)$ are in the right half plane, so the poles of $G(z)$ are all on the imaginary axis (Lemma 2 ). Substituting (18) into Eq. (3) we see that

$$
\widetilde{G}(z) G(z)=\frac{\left(1+z^{-1}\right)^{2 N}}{\left(1+z^{-1}\right)^{2 N}+(-1)^{N}\left(1-z^{-1}\right)^{2 N}}
$$

For example if $N=1$ this reduces to

$$
\widetilde{G}(z) G(z)=\frac{z+2+z^{-1}}{4}
$$

which yields the spectral factor $G(z)=\left(1+z^{-1}\right) / 2$. The first order Butterworth power symmetric filter therefore reduces to an FIR filter. For $N=2$ we have

$$
\widetilde{G}(z) G(z)=\frac{\left(1+z^{-1}\right)^{4}}{2\left(1+6 z^{-2}+z^{-4}\right)}
$$

from which we can identify a causal stable spectral factor

$$
G(z)=\frac{\left(1+z^{-1}\right)^{2}}{\sqrt{2}\left(1+\rho z^{-2}\right)}
$$

where $\rho=3-\sqrt{8} \approx 0.1716$. This is the second order Butterworth power symmetric filter. The matlab command butter $(N, 0.5)$ produces a power symmetric Butterworth filter of order $N$, and the denominator is a function of $z^{-2}$. For $N=9$ the magnitude response is shown in Fig. 3 .

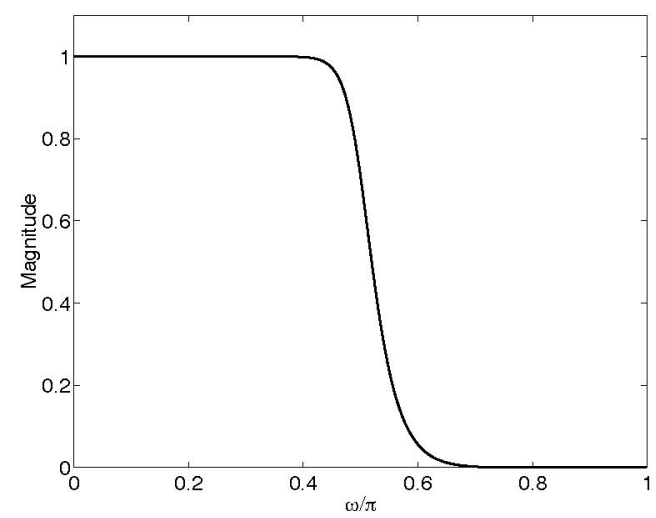

Figure 3. Magnitude response of the 9 th order IIR power symmetric Butterworth filter.

The transfer function is

$$
G(z)=\frac{c\left(1+z^{-1}\right)^{9}}{D(z)}
$$

where $c$ is a nonzero constant. Furthermore, since $G(z)$ satisfies the conditions of Theorem 5.3.1 in [5], it can be written as a sum of two allpass polyphase components:

$G(z)=\frac{a_{0}\left(z^{2}\right)+z^{-1} a_{1}\left(z^{2}\right)}{2}=\frac{z^{-4} \beta_{0}\left(z^{-2}\right)}{2 \beta_{0}\left(z^{2}\right)}+\frac{z^{-5} \beta_{1}\left(z^{-2}\right)}{2 \beta_{1}\left(z^{2}\right)}$

where

$$
\beta_{0}(z)=1+0.3644 z^{-1}+0.0104 z^{-2}
$$

and

$$
\beta_{1}(z)=1+0.8366 z^{-1}+0.09336 z^{-2} .
$$

\section{CONCLUding REMARKS}

One question still remains. Is the converse of Lemma 2 true? That is, if a power symmetric filter has all its poles on the imaginary axis, then does it imply that $R(z)$ has all its poles located entirely on one side of the imaginary axis? The answer is in the negative, as shown by counter examples. Thus consider the power symmetric filter $G(z)$ given by

$$
\frac{1}{2}\left(\frac{0.7+z^{-2}}{1+0.7 z^{-2}}\right)\left(\frac{0.9+z^{-2}}{1+0.9 z^{-2}}\right)+\frac{z^{-1}}{2}\left(\frac{0.2+z^{-2}}{1+0.2 z^{-2}}\right)
$$

The poles are on the imaginary axis of the $z$-plane at the points

$$
\pm j \sqrt{0.2}, \pm j \sqrt{0.7}, \pm j \sqrt{0.9}
$$

The poles of $\widetilde{R}(z) R(z)$ can be computed from here, and it can be verified that two of these poles are at the locations $z=-1$ and

$$
z=0.1364+0.7348 j
$$

(or its reciprocal conjugate location). Thus the poles of $R(z)$ are not restricted to be in one half-plane though $G(z)$ has all poles on the imaginary axis.

While the results presented in this paper have mostly been theoretical, a general study of power symmetric filters has not been presented earlier in the literature. Since power symmetric filters generate orthonormal bases (when used in filter banks) these results can be of interest in situations where such IIR bases have to be generated efficiently.

\section{REFERENCES}

[1] A. Antoniou Digital signal processing, McGraw-Hill, 2006.

[2] A. V. Oppenheim and R. W. Schafer, Digital signal processing, Prentice Hall, Inc., 1975.

[3] D. J. Rabideau and P. Parker, "Ubiquitous MIMO multifunction digital array radar", IEEE Asilomar conference on signals, systems, and computers, Pacific Grove, CA, 2003.

[4] T.A. Ramstad, "IIR filterbank for subband coding of images," Proc. of the IEEE Int. Symp. on Circuits and Systems, pp. 827830, Finland, June 1988.

[5] P. P. Vaidyanathan, Multirate systems and filter banks, Prentice Hall, Inc., 1993.

[6] P. P. Vaidyanathan, S. K. Mitra, Y. Neuvo, "A new approach to the realization of low sensitivity IIR digital filters," IEEE Trans. on Acoustics, Speech and Signal Processing, pp. 350-361, April 1986.

[7] Vetterli, M., and Herley, C. "Wavelets and filter banks," IEEE Trans. on Signal Processing, vol. SP-40, 1992. 